\title{
Galaktografie mit Tomosynthese (Galaktomosynthese) - Renaissance einer Methode?
}

\section{Galactography with Tomosynthesis Technique (Galactomosynthesis) - Renaissance of a Method?}

(c) 9 (9)

\author{
Autoren \\ Institute \\ 1 Radiologisches Institut, Universitätsklinikum Erlangen, \\ CCC ER-EMN, Universitäts-Brustzentrum Franken, Erlangen \\ 2 Frauenklinik, Universitätsklinikum Erlangen, CCC ER-EMN, \\ Universitäts-Brustzentrum Franken, Erlangen \\ 3 Klinik für Radiologie - Klinikum Frankfurt Hoechst, \\ DKG-zertifiziertes Brustzentrum, Frankfurt am Main
}

Rüdiger Schulz-Wendtland ${ }^{1}$, Caroline Preuss ${ }^{2 *}$, Peter A. Fasching ${ }^{2}$, Christian R. Loehberg ${ }^{2}$, Michael P. Lux ${ }^{2}$, Julius Emons ${ }^{2}$, Matthias W. Beckmann², Michael Uder ${ }^{1}$, Markus Mueller-Schimpfle ${ }^{3}$

Schlüsselwörter

Mamma, Galaktografie, Tomosynthese, Galaktomosynthese, Ultraschall, MRT

Key words

breast, galactography, tomosynthesis, galactomosynthesis, ultrasound, MRI

eingereicht 21.2.2018

revidiert 12.3 .2018

akzeptiert 18.3.2018

Bibliografie

DOI https://doi.org/10.1055/a-0594-2277

Geburtsh Frauenheilk 2018; 78: 493-498 @ Georg Thieme

Verlag KG Stuttgart · New York | ISSN 0016-5751

Korrespondenzadresse

Prof. Dr. med. R. Schulz-Wendtland

Universitätsklinikum Erlangen, Radiologisches Institut/

Gynäkologische Radiologie

Universitätsstraße 21-23, 91054 Erlangen

ruediger.schulz-wendtland@uk-erlangen.de

\section{ZUSAMMENFASSUNG}

Einleitung Die konventionelle Galaktografie stellte jahrzehntelang das einzige bildgebende Verfahren zur Darstellung von Milchgängen in der Brust dar. Heute verfügen wir in der Diagnostik über ein multimodales Konzept aus hochauflösendem Ultraschall, der Magnetresonanz-(MR-)Mammografie und der Duktoskopie/Galaktoskopie mit Sensitivitäten und Spezifitäten bis zu 95\%. Ziel unserer Untersuchung war es, erstmalig die Tomosynthesetechnik in der Galaktografie einzusetzen und die daraus generierten synthetischen digitalen 2-D-Vollfeld-Mammografien mit der etablierten Methode der duktusorientierten Sonografie zu vergleichen. Es sollen mit beiden Methoden invasive Mammakarzinome und deren Vorstufen wie duktale Carcinoma in situ (DCIS) sowie benigne Befunde erkannt werden.

Material und Methoden Wir führten bei 5 Patientinnen mit pathologischer Mamillensekretion sowohl eine duktusorientierte Sonografie, eine kontrastmittelunterstützte Galaktografie mithilfe der Tomosynthese in 3-D sowie auch den daraus generierten synthetischen digitalen 2-D-VollfeldMammografien durch. Die Auswertung der unterschiedlichen Untersuchungsmodalitäten erfolgte durch 3 in der komplementären Mammadiagnostik erfahrene Untersucher (1, 5 und 15 Jahre) und wurde mit der endgültigen Histologie korreliert.

Ergebnisse Alle 3 Untersucher beurteilten unabhängig voneinander die Bilder des duktusorientierten Ultraschalls und der kontrastmittelunterstützten Galaktografie in Tomosynthesetechnik in 3-D und den daraus generierten, synthetischen digitalen 2-D-Vollfeld-Mammografien. Die Ergebnisse wurden mit den histopathologischen Befunden der Operationspräparate korreliert, wobei sich bei den 5 Patientinnen 1 invasives Mammakarzinom, 2-mal ein duktales Carcinoma in situ (DCIS) und 2 benigne Befunde ergaben. Alle 3 Untersucher lagen bei der Verdachtsdiagnose in der Standardbildgebung der duktusorientierten Sonografie seltener richtig als bei der erstmalig durchgeführten, kontrastmittelunterstützten Galaktografie in Tomosynthesetechnik und den daraus generierten, synthetischen digitalen 2-D-Vollfeld-Mammografien.

* geteilte Erstautorenschaft 
Schlussfolgerung Erstmalig wurde die Brusttomosynthese in der Galaktografie (Galaktomosynthese) eingesetzt und ermöglichte eine digitale, 3-dimensionale Darstellung von suspekten Befunden. Zusammen mit den daraus synthetisierten, digitalen 2-D-Vollfeld-Mammografien könnte dies in Zukunft eine sinnvolle Ergänzung der komplementären Mammadiagnostik sein - und eine Renaissance dieser Methode. Im Vergleich mit dem duktusorientierten Ultraschall in Hochauflösung erzielten die Untersucher mit der kontrastmittelunterstützten Galaktografie in Tomosynthesetechnik und den daraus generierten, synthetischen digitalen 2-D-Vollfeld-Mammografien bessere Ergebnisse in Korrelation mit den histopathologischen Befunden.

\section{ABSTRACT}

Introduction For decades, conventional galactography was the only imaging technique capable of showing the mammary ducts. Today, diagnosis is based on a multimodal concept which combines high-resolution ultrasound with magnetic resonance (MR) mammography and ductoscopy/galactoscopy and has a sensitivity and specificity of up to $95 \%$. This study used tomosynthesis in galactography for the first time and compared the synthetic digital 2D full-field mammograms generated with this technique with the images created using the established method of ductal sonography. Both methods should be able to detect invasive breast cancers and their precursors such as ductal carcinoma in situ (DCIS) as well as being able to identify benign findings.

Material and Methods Five patients with pathological nipple discharge were examined using ductal sonography, contrast-enhanced 3D galactography with tomosynthesis and the synthetic digital 2D full-field mammograms generated with the latter method. Evaluation of the images created with the different imaging modalities was done by three investigators with varying levels of experience with complementary breast diagnostics (1, 5 and 15 years), and their evaluations were compared with the histological findings.

Results All 3 investigators independently evaluated the images created with ductal sonography, contrast-enhanced 3D galactography with tomosynthesis, and generated synthetic digital 2D full-field mammograms. Their evaluations were compared with the histopathological assessment of the surgical specimens resected from the 5 patients. There was 1 case of invasive breast cancer, 2 cases with ductal carcinoma in situ and 2 cases with benign findings. All 3 investigators made more mistakes when they used the standard imaging technique of ductal sonography to diagnose suspicious lesions than when they used contrast-enhanced galactography with tomosynthesis and the generated synthetic digital 2D fullfield mammograms.

Conclusion This is the first time breast tomosynthesis was used in galactography (galactomosynthesis) to create digital 3-dimensional images of suspicious findings. When used together with the generated synthetic digital 2D full-field mammograms, it could be a useful complementary procedure for the diagnosis of breast anomalies and could herald a renaissance of this method. Compared with high-resolution ductal ultrasound, the investigators achieved better results with contrast-enhanced galactography using tomosynthesis and the generated synthetic digital 2D full-field mammograms, as confirmed by histopathological findings.

\section{Einleitung}

Die konventionelle Galaktografie wurde bereits in den 1930er-Jahren von E. Ries beschrieben. Das dabei verwendete Lipiodol, das in sezernierende Milchgänge injiziert wurde, barg zu viele Komplikationen in Form von Entzündungen und Abszessbildungen, da das ölige Kontrastmittel schlecht resorbiert und ausgeschieden wurde. Mit dem Einsatz wasserlöslicher Kontrastmittel in den 1940er-jahren gelangen erste zweckmäßige diagnostische Ergebnisse [1,2]. In den 1960er- und 1970er-Jahren wurde die Galaktografie erstmals in größeren Studien erprobt und zunehmend in der klinischen Routine eingesetzt [1,2]. Die grundlegende Technik hat sich seitdem kaum verändert [29]. Duktektasien, Füllungsdefekte oder Gangabbrüche weisen indirekt auf eine vorliegende Pathologie hin, lassen prinzipiell aber keine Artdiagnose zu und müssen histopathologisch abgeklärt werden [16,29]. Zwischenzeitlich konnte die Galaktografie von den Fortschritten in der Röntgentechnik und der Anwendung von Lokalanästhetika sowie weiterentwickelter, nichtionischer Kontrastmittel profitieren.

Jahrzehntelang war dennoch die konventionelle Galaktografie (Sensitivitäten und Spezifitäten bis zu 95\%) [1-16] das einzige bildgebende Verfahren zur Darstellung von Milchgängen der Brust.
Heute steht uns neben der konventionellen Galaktografie ein multimodales Konzept zur Verfügung: Ultraschall mit hochfrequenten Schallsonden (10-18 MHz) (Sensitivitäten und Spezifitäten bis zu 91\%) $[8,10,12,13,15,17-21]$, die Magnetresonanz(MR-)Mammografie (Sensitivitäten und Spezifitäten bis zu $89 \%$ bzw. 93\%) [5, 13, 14, 19, 21 - 23] sowie die Duktoskopie (Sensitivitäten und Spezifitäten bis zu 71 bzw. 62\%) [24-26]. Insbesondere die Sonografie (Sensitivitäten und Spezifitäten bis zu 91\%) [8, 10, $12,13,15,17-21$ ] ist mittlerweile in allen Brustzentren verfügbar, und durch den Gebrauch hochfrequenter Schallsonden (10$18 \mathrm{MHz}$ ) ist auch eine qualitätsorientierte Duktussonografie möglich. Daneben bietet die Sonografie bei pathologischen Befunden (BI-RADS'M 4/5) auch die Möglichkeit einer einfachen, kostengünstigen, bildgesteuerten Biopsie. Die MR-Mammografie (Sensitivitäten und Spezifitäten bis zu 89 bzw. 93\%) [5, 13, 14, 19, 21 23] findet zunehmend Anwendung, da auch in vielen Zentren MR-gesteuerte Biopsien möglich sind. Zusätzlich ergänzt wird das Spektrum der Diagnostik durch die Durchführung einer Duktuskopie/Galaktoskopie (Sensitivitäten und Spezifitäten bis zu 71 bzw. 62\%) [24-26] mit gleichzeitiger histologischer Verifizierung der Befunde.

Die digitale Vollfeldmammografie (Full-Field Digital Mammography [FFDM]) ist das wichtigste Verfahren zur Frühdiagnose des 
Mammakarzinoms bzw. des duktalen Carcinoma in situ (DCIS). Die für ein Projektionsverfahren typische Überlagerung von Gewebestrukturen mindert jedoch häufig Spezifität und Sensitivität der mammografischen Untersuchung. Die digitale Tomosynthese der Brust (Digital Breast Tomosynthesis [DBT]) liefert auf der Basis einer begrenzten Anzahl von Einzelaufnahmen mit unterschiedlichen Projektionswinkeln überlagerungsfreie Schichtaufnahmen und kann so die unerwünschte Maskierung durch überlagerte Strukturen beseitigen. Klinische Untersuchungen zeigen, dass die digitale Tomosynthese tatsächlich das Potenzial besitzt, die Wiedereinbestellrate von Patienten zu reduzieren und gleichzeitig die Karzinomentdeckung zu steigern (Detektion und Volumetrie) - dies vor allem bei Frauen mit dichtem Brustgewebe [27, 30].

Ziel der vorliegenden Untersuchung war es, erstmalig die Tomosynthesetechnik [27] in der Galaktografie einzusetzen und die daraus generierten synthetischen digitalen 2-D-Vollfeld-Mammografien [28] mit der etablierten Methode der duktusorientierten Sonografie zu vergleichen. Es sollen mit beiden Methoden invasive Mammakarzinome und deren Vorstufen wie duktale Carcinoma in situ (DCIS) sowie benigne Befunde erkannt werden.

\section{Material und Methoden}

\section{Patientinnenkollektiv}

Am Radiologischen Institut des Universitätsklinikums Erlangen und der Klinik für Radiologie - Klinikum Frankfurt Hoechst wurde bei insgesamt 5 Patientinnen neben der routinemäßigen komplementären Mammadiagnostik bestehend aus klinischer Untersuchung, Mammasonografie und Mammografie zusätzlich sowohl eine duktusorientierte Sonografie als auch eine kontrastmittelunterstützte Galaktografie mithilfe der Tomosynthese in 3-D- als auch den daraus generierten synthetischen digitalen 2-D-Vollfeld-Mammografien durchgeführt.

Einschlusskriterium zur Studienteilnahme war die einseitige pathologische Sekretion: blutige Sekretion oder spontane klar/seröse, trübe oder bräunlich/grüne (nicht milchige) Sekretion. Ausschlusskriterium waren Patientinnen mit voroperierter Mamma, Zustand nach Bestrahlung der Mamma oder medikamentöser Vorbehandlung.

\section{Duktusorientierte Sonografie, Galaktografie, Brusttomosynthese}

Der duktusorientierte Ultraschall wurde zunächst mit einem hochauflösenden Ultraschallsystem (12-15 MHz), elektronischer Focus, linear-array Transducer (Siemens ${ }^{\mathrm{TM}}$, Acuson, Erlangen, Germany) durchgeführt. Die Beurteilungskriterien waren: unauffälliger Milchgang, Duktektasien, suspekter intraduktaler Befund. Bei allen Patientinnen erfolgte zusätzlich eine Galaktografie: Nach Lokalanästhesie und Aufsuchen des betroffenen Milchausführungsganges erfolgte nach Positionierung der Kanüle (Fa. Cook, Baesweiler, 30-G-Sialografie-Nadel) die Applikation von 0,1 bis zu $1,5 \mathrm{ml}$ wasserlöslichem Kontrastmittel. Anschließend wurde der Ausführungsgang mithilfe eines Tupfers bzw. Pflasters komprimiert und mit einem Sprayverband versehen, um ein Austreten des Kontrastmittels zu verhindern [29]. Im Anschluss dieser Untersuchung wurden bei jeder Patientin eine digitale Brust- tomosynthese und die daraus berechneten synthetischen digitalen 2-D-Vollfeld-Mammografieaufnahmen erstellt [27-28]. Bei den mammografischen Systemen handelte es sich um:

1. Selenia Dimensions (Hologic Bedfort ${ }^{\mathrm{TM}}$ MA, USA) und

2. Amulet Innovality (Fujifilm ${ }^{\mathrm{TM}}$, Deutschland).

Die Tomosynthese erfolgte bei dem Selenia-System in einer Ebene mit einem Rotationswinkel $\pm 7,5$ Grad, Pixelgröße $70 \mu \mathrm{m}$, räumliche Aufteilung 8 Linienpaare (Lp)/mm bzw. bei dem Amulet Innovality System mit einem Standard-Rotationswinkel von \pm 7,5 Grad (hochauflösend von \pm 20 Grad), Pixelgröße 150/100 $\mu \mathrm{m}$ (100/ $50 \mu \mathrm{m})$, räumliche Auflösung $3 \mathrm{Lp} / \mathrm{mm} ; 5$ Lp/mm bzw. $10 \mathrm{Lp} /$ $\mathrm{mm}$. Die Dosis entsprach in der Engwinkeltechnik der einer digitalen 2-D-Vollfeld-Mammografie von im Durchschnitt $1,70 \mathrm{mSv}$ (Standardabweichung 0,42) und damit unterhalb der gesetzlichen Vorgabe von maximal 2,5 mGy/Aufnahme [29]. Die Aufnahmezeit betrug im Mittelwert 10 Sekunden. Mithilfe der Prozesssoftware der jeweiligen Systeme wurden aus den Tomosynthesedaten die synthetischen digitalen 2-D-Vollfeld-Mammografieaufnahmen generiert. Die Beurteilungskriterien waren sowohl für die Tomosynthese als auch die aus den Tomosynthesesequenzen generierten synthetischen digitalen 2-D-Vollfeld-Mammografien: Normalbefund, Duktektasien, singulärer Füllungseffekt, multiple Füllungsdefekte und Gangabbruch [16].

\section{Operation und Pathologie}

Alle Patientinnen wurden nach entsprechender Hautmarkierung zunächst duktektomiert. Jedes Operationspräparat wurde histopathologisch aufgearbeitet. Bei dem invasiven Mammakarzinom erfolgte in einem 2. operativen Eingriff die brusterhaltende Therapie mit Sentinellymphknotenbiopsie (SLN).

\section{Statistische Analyse}

Die Auswertung der 3 Untersuchungsmodalitäten erfolgte durch 3 in der komplementären Mammadiagnostik erfahrene Untersucher (1, 5 und 15 Jahre) und wurde mit der endgültigen Histologie korreliert. Bei sehr kleiner Fallzahl erschien die Berechnung von Sensitivität, Spezifität, dem ppV als Anzahl der richtig positiven/(Anzahl der richtig positiven + falsch positiven Ergebnisse) sowie des npV als Anzahl der richtig negativen/(Anzahl der richtig negativen + Anzahl der falsch negativen Ergebnisse) nicht sinnvoll.

\section{Ergebnisse}

Es wurde bei insgesamt 5 Patientinnen ein duktusorientierter Ultraschall und im Anschluss eine 3-D-digitale Tomosynthese ( $\vee$ Abb. 1 a und $\mathbf{b}$ ) und den aus den Datensätzen generierten synthetischen digitalen 2-D-Vollfeld-Mammografien als Galaktografie ( $\triangleright$ Abb. 1 c) durchgeführt. Drei Untersucher (1, 5, 15 Jahre Berufserfahrung) werteten unabhängig voneinander die in der Bildgebung dargestellten Befunde aus.

Das mittlere Alter der Patientinnen betrug 54,2 Jahre (minimal 21 Jahre, maximal 83 Jahre). 

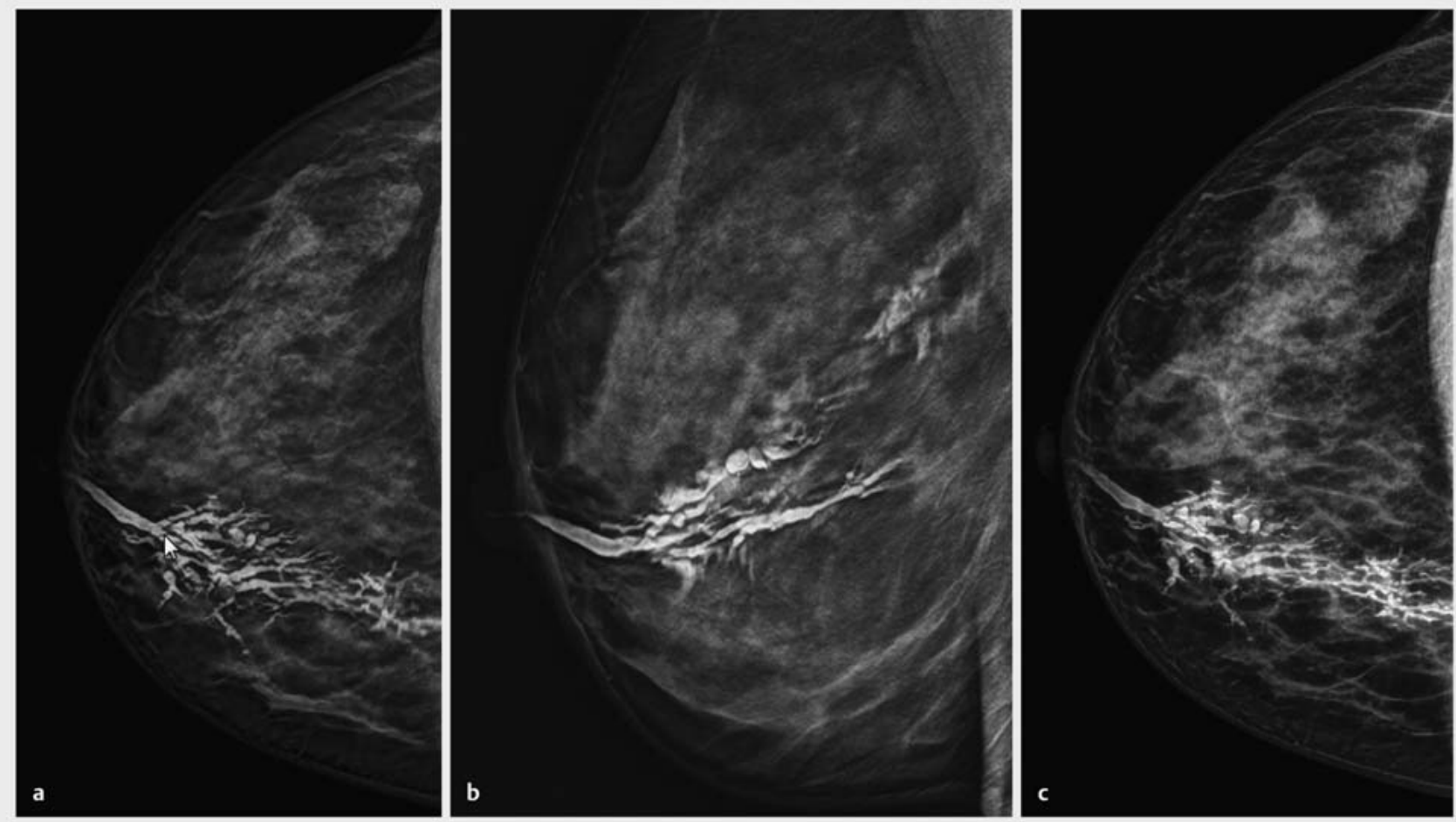

- Abb. 1 a und b Kontrastmittelunterstützte Galaktografie in Tomosynthesetechnik in 3-D (DBT). a Einzelbild in CC-Projektion rechte Mamma; b Einzelbild in ML-Projektion rechte Mamma. c Aus der kontrastmittelunterstützten Galaktografie in Tomosynthesetechnik (3-D) generierte, synthetische, digitale 2-D-Vollfeld-Mammografie in CC-Projektion der Abb. a; Diagnose: multiple Füllungsdefekte und Gangabbrüche - BIRADSTM 4 [31]; Histologie: ausgedehnte Papillomatose der rechten Mamma.

\section{Histopathologischer Befund}

Histologisch ergaben sich bei den OP-Präparaten der 5 Patientinnen 1 invasives Mammakarzinom, 2-mal ein duktales Carcinoma in situ (DCIS) und 2 benigne Befunde als endgültige Diagnosen.

\section{Vergleich zwischen duktusorientiertem Ultraschall und Brusttomosynthese mit Galaktografie und den daraus synthetisierten digitalen 2-D-Vollfeld- Mammografien}

Untersucher 1 mit 1-jähriger Berufserfahrung in der komplementären Mammadiagnostik erkannte in dem duktusorientierten hochfrequenten Ultraschall ( $\triangleright$ Abb. 2) 3 von 5 Diagnosen und lag 2-mal falsch - demgegenüber stellte er anhand der Brusttomosynthese und den daraus synthetisch erstellten digitalen 2-D-Vollfeld-Mammografien die Diagnose 4 von 5 mal richtig.

Untersucher 2 mit 5 Jahren Berufserfahrung lag bei der Beurteilung des duktusorientierten Ultraschalls ebenfalls bei 3 von 5 Diagnosen richtig - in der Brusttomosynthese und den daraus synthetisch erstellten digitalen 2-D-Vollfeld-Mammografien stimmten 5 von 5 Diagnosen überein.

Untersucher 3 mit 15 Jahren Berufserfahrung in der komplementären Mammadiagnostik erkannte in der duktusorientierten Sonografie 4 von 5 Befunden korrekt. Mittels der Brusttomosynthese und den daraus synthetisch erstellten digitalen 2-D-Voll-
feld-Mammografien wurde die Diagnose 5 von 5 mal korrekt gestellt bezogen auf die endgültige Histologie.

Auf die Angabe von Sensitivität, Spezifität, positivem prädikiven Wert und negativem prädiktiven Wert wird aufgrund der kleinen Fallzahl verzichtet.

\section{Diskussion}

Wir konnten mit unserer Untersuchung erstmalig zeigen, dass die Brusttomosynthese auch in der Galaktografie (Galaktomosynthese) einsetzbar ist und eine digitale, 3-dimensionale Darstellung suspekter Befunde ermöglicht. Zusammen mit den daraus generierten synthetischen digitalen 2-D-Vollfeld-Mammografien bietet sich eine zusätzliche Option in der Mammadiagnostik.

Zudem führten wir einen Vergleich zwischen der etablierten diagnostischen Methode der duktusorientierten, hochfrequenten Sonografie (12-15 MHz) und der kontrastmittelunterstützten Galaktografie in Tomosynthesetechnik und den daraus generierten synthetischen digitalen 2-D-Vollfeld-Mammografien durch.

Die Auswertungen zeigen eine annähernde Gleichwertigkeit von duktusorientiertem hochfrequenten Ultraschall und den aus der kontrastmittelunterstützten Galaktografie in Tomosynthesetechnik generierten, synthetischen digitalen 2-D-Vollfeld-Mammografien in Hinsicht auf die richtig gestellte Verdachtsdiagnose und die endgültigen histopathologischen Befunde. Auf die Anga- 


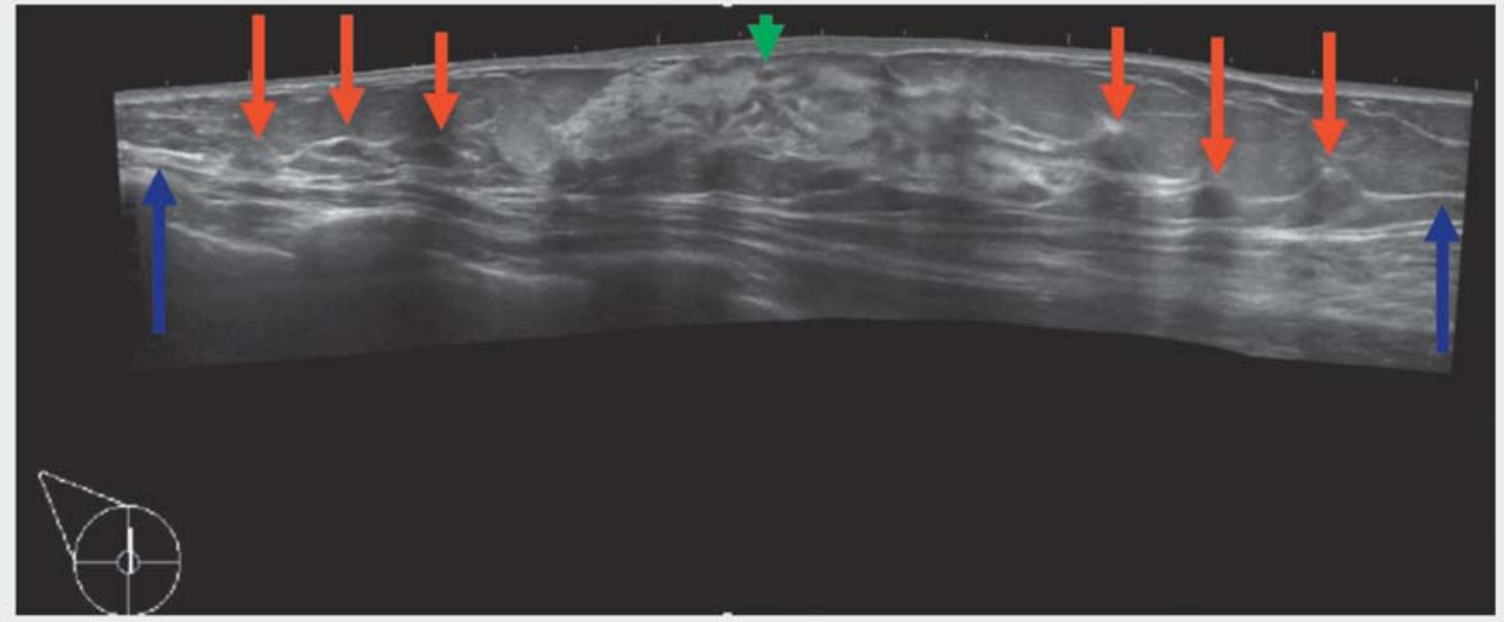

- Abb. 2 Hochauflösender duktusorientierter Ultraschall (12-15 MHz). Multiple Papillome mit Gangabbrüchen bei blutig sezernierendem Milchgang (blau: Gangabrüche, rot: multiple Papillome, grün: Mamille).

be von Sensitivität, Spezifität, positivem prädiktiven Wert und negativem prädiktiven Wert wird in dieser Untersuchung aufgrund der kleinen Fallzahl verzichtet.

Bisher existiert keine Literatur zur kontrastmittelunterstützten Galaktografie in Tomosynthesetechnik und den daraus generierten synthetischen digitalen 2-D-Vollfeld-Mammografien.

Insgesamt ergibt unsere Untersuchung, dass mehr richtige Verdachtsdiagnosen in Hinsicht auf die endgültige Histologie anhand der kontrastmittelunterstützten Galaktografie in Tomosynthesetechnik und den daraus generierten synthetischen digitalen 2-D-Vollfeld-Mammografien erlangt wurden als durch die Standarddiagnostik des duktusorientierten hochfrequenten Ultraschalls. Das Ergebnis trifft auf alle 3 Untersucher zu, die jeweils unterschiedlich lange Berufserfahrung im Bereich der komplementären Mammadiagnostik (1, 5, 15 Jahre) besitzen ( $\bullet$ Tab. $\mathbf{1})$.

Das Ergebnis ist insofern überraschend, als dass die Brusttomosynthese und im Speziellen die kontrastmittelunterstützte Galaktografie in Tomosynthesetechnik keine Routinetechniken in der Mammadiagnostik sind. Demgegenüber werden die Mammasonografie und der duktusorientierte Ultraschall als Routinediagnostik regelmäßig eingesetzt und die Untersucher weisen in deren Beurteilung einen hohen Erfahrungsschatz auf. Ein Vergleich mit Literaturdaten ist hier nicht möglich, da keine vergleichende Literatur verfügbar ist.

Limitationen unserer Untersuchung sind vor allem in der beschränkten Fallzahl zu sehen. Dennoch können durch unsere Daten Fragen und Hypothesen aufgeworfen werden:

1. Erstmaliger Einsatz der Tomosynthese in der Galaktografie (Galaktomosynthese) mit tendenziell besseren Ergebnissen im Vergleich zum duktusorientierten hochfrequenten Ultraschall im Sinne eines Proof-of-concept - kann dies zu einer Renaissance der Galaktografie führen?

2. Erweiterung des Spektrums der Tomosynthese in der komplementären Mammadiagnostik.

In einer Literaturdurchsicht (PubMed) unter den Stichworten: Breast, Mammography, Ultrasound, Galactography, MRI, Ductography, Tomosynthesis wird deutlich, dass bisher keine vergleichende Untersuchung von klinischer Untersuchung, Abstrichzytologie, duktusorientiertem hochauflösendem Ultraschall, Galaktografie, MR-Mammografie, Duktoskopie mit histologischer Korrelation oder gar der Galaktografie mit DBT (Galaktomosynthese) existiert.

- Tab. 1 Ergebnisse der 3 Untersucher: Anzahl richtiger Verdachtsdiagnosen im Hinsicht auf die endgültige Histologie der Präparate $(n=5)$ in Abhängigkeit der Bildmodalität.

\begin{tabular}{|l|l|l|}
\hline Bildmodalität & $\begin{array}{l}\text { duktusorientierter hochfrequenter } \\
\text { Ultraschall (12-15 } \mathbf{M H z})\end{array}$ & $\begin{array}{l}\text { kontrastmittelunterstützte Galaktografie in } \\
\text { Tomosynthesetechnik (3-D) und daraus generierte, } \\
\text { synthetische digitale 2-D-Vollfeld-Mammografie }\end{array}$ \\
\hline Untersucher 1 (1 Jahr Beruferfahrung) & 3 & 4 \\
\hline Untersucher 2 (5 Jahre Berufserfahrung) & 3 & 5 \\
\hline Untersucher 3 (15 Jahre Berufserfahrung) & 4 & 5 \\
\hline
\end{tabular}


Mit unserer Untersuchung konnten wir jedoch erste interessante Ergebnisse erzielen. Ziel sollte sein, diese Ergebnisse in einer multizentrischen Studie zu evaluieren. Sollten sich die von uns erzielten Studienergebnisse auch an einem größeren Patientenkollektiv bestätigen lassen, könnte die Tomosynthese und insbesondere die kontrastmittelunterstützte Galaktografie in Tomosynthesetechnik (Galaktomosynthese) mit den daraus generierten synthetischen digitalen 2-D-Vollfeld-Mammografien die komplementäre Mammadiagnostik sinnvoll ergänzen und zu einer Renaissance dieser Methode führen.

Durch unsere Methodik wird es möglich sein, einerseits die Anzahl unnötiger Operationen zu reduzieren und andererseits das operative Management durch die 3-dimensionale Visualisierung des pathologischen Prozesses zu optimieren.

Interessenkonflikt

Die Autoren geben an, dass kein Interessenkonflikt besteht.

Literatur

[1] Scheurlen K, Schnitzer A, Krammer J et al. Stellenwert der Galaktographie zur Abklärung pathologischer Sekretion in der komplementären Mammadiagnostik - Teil 1: Eine Onlineumfrage an den deutschen Brustzentren. Radiologe 2014; 54: 63-67

[2] Scheurlen K, Schnitzer A, Krammer J et al. Stellenwert der Galaktographie zur Abklärung pathologischer Sekretion in der komplementären Mammadiagnostik - Teil 2: Eine systematische Literaturanalyse. Radiologe 2014; 54: 160-166

[3] Ciatto S, Bravetti P, Berni D et al. The role of galactography in the detection of breast cancer. Tumori 1988; 74: 177-181

[4] Grillo ME, Lehmann-Willenbrock E, Gent H]. Chromogalactography preceding ductal-lobular unit excision for nipple discharge-with special reference to diagnostic galactography and histology. Ann Chir Gynaecol 1990; 79: 6-9

[5] Krämer SC, Rieber A, Gorich J et al. Diagnosis of papillomas of the breast: value of magnetic resonance mammography in comparison with galactography. Eur Radiol 2000; 10: 1733-1736

[6] Dinkel HP, Gassel AM, Müller T et al. Galactography and exfoliative cytology in women with abnormal nipple discharge. Obstet Gynecol 2001; 97: 625-629

[7] Baitchev G, Gortchev G, Todorova A et al. Intraductal aspiration cytology and galactography for nipple discharge. Int Surg 2003; 88: 83-86

[8] Cabioglu N, Hunt KK, Singletary SE et al. Surgical decision making and factors determining a diagnosis of breast carcinoma in women presenting with nipple discharge. J Am Coll Surg 2003; 196: 354-364

[9] Funovics MA, Phillipp MO, Lackner B et al. Galactography: method of choice in pathologic nipple discharge? Eur Radiol 2003; 13: 94-99

[10] Adepoju LJ, Chun J, El-Tamer M et al. The value of clinical characteristics and breast-imaging studies in predicting a histopathologic diagnosis of cancer or high-risk lesion in patients with spontaneous nipple discharge. Am J Surg 2005; 190: 644-646

[11] Hünerbein M, Raubach M, Gebauer B et al. Ductoscopy and intraductal vacuum assisted biopsy in women with pathologic nipple discharge. Breast Cancer Res Treat 2006; 99: 301-307
[12] Gray R], Pockaj BA, Karstaedt PJ. Navigating murky waters: a modern treatment algorithm for nipple discharge. Am J Surg 2007; 194: 850854

[13] Grunwald S, Heyer H, Paepke S et al. Diagnostic value of ductoscopy in the diagnosis of nipple discharge and intraductal proliferations in comparison to standard methods. Onkologie 2007; 30: 243-248

[14] Morrogh M, Morris EA, Liberman L et al. The predictive value of ductography and magnetic resonance imaging in the management of nipple discharge. Ann Surg Oncol 2007; 14: 3369-3377

[15] Hamed ST, Abdo MH, Ahmed HH. Breast discharge: ultrasound and Doppler evaluation. J Egypt Natl Canc Inst 2008; 20: 262-270

[16] Montroni I, Santini D, Zucchini G et al. Nipple discharge: is its significance as a risk factor for breast cancer fully understood? Observational study including 915 consecutive patients who underwent selective duct excision. Breast Cancer Res Treat 2010; 123: 895-900

[17] Grunwald S, Bojahr B, Schwesinger G et al. Mammary ductoscopy for the evaluation of nipple discharge and comparison with standard diagnostic techniques. J Minim Invasive Gynecol 2006; 13: 418-423

[18] Vargas HI, Vargas MP, Eldrageely K et al. Outcomes of clinical and surgical assessment of women with pathological nipple discharge. Am Surg 2006; 72: 124-128

[19] Choudhury S, Isomoto I, Hyashi K. Magnetic resonance imaging features of breasts in patients with nipple discharge. Bangladesh Med Res Counc Bull 2008; 34: 44-47

[20] Liu GY, Lu JS, Shen KW et al. Fiberoptic ductoscopy combined with cytology testing in the patients of spontaneous nipple discharge. Breast Cancer Res Treat 2008; 108: 271-277

[21] Morrogh M, Park A, Elkin EB et al. Lessons learned from 416 cases of nipple discharge of the breast. Am J Surg 2010; 200: 73-80

[22] Schwab SA, Uder M, Schulz-Wendtland R et al. Direct MR galactography: feasibility study. Radiology 2008; 249: 54-61

[23] Wenkel E, Janka R, Uder M et al. Does direct MR galactography have the potential to become an alternative diagnostic tool in patients with pathological nipple discharge? Clin Imaging 2011; 35: 85-93

[24] Ohlinger R, Stomps A, Paepke S et al. Ductoscopic detection of intraductal lesions in cases of pathologic nipple discharge in comparison with standard diagnostics: the German multicenter study. Oncol Res Treat 2014; 37: 628-632

[25] Schulz-Wendtland R, Aichinger U, Krämer S et al. [Galactoscopy-is it a new interventional method for breast diagnosis?]. Rofo 2002; 174 : 1015-1017

[26] Schulz-Wendtland R, Meier-Meitinger M, Adamietz B et al. Digitale Galaktoskopie der 2. Generation. Geburtsh Frauenheilk 2011; 71: 255-259

[27] Schulz-Wendtland R, Beckmann MW, Fasching PA et al. Tomosynthese. Gynäkol Prax 2015; 39: 521-534

[28] Choi JS, Han BK, Ko EY et al. Comparison between two-dimensional synthetic mammography reconstructed from digital breast tomosynthesis and full-field digital mammography for the detection of $\mathrm{T} 1$ breast cancer. Eur Radiol 2016. doi:10.1007/s00330-015-4083-7

[29] S3-Leitlinie Früherkennung, Diagnose, Therapie und Nachsorge des Mammakarzinoms, Version 4.0. 2017. AWMF Registernummer: 032045OL. Online: http://www.leitlinienprogramm-onkologie.de/leitlinien/ mammakarzinom/; Stand: 12.02.2018

[30] Rafferty EA, Durand MA, Conant EF et al. Breast cancer screening using tomosynthesis and digital mammography in dense and nondense breasts. JAMA 2016; 315: 1784-1786

[31] Morris EA, Comstock C, Lee C et al. ACR BI-RADSTM - Atlas der Mammadiagnostik. Berlin, Heidelberg: Springer-Verlag; 2016 